\title{
Dance Experience and Associations with Cortical Gray Matter Thickness in the Aging Population
}

\author{
Shai Porat ${ }^{a, b}$ Naira Goukasian ${ }^{a, b}$ Kristy S. Hwang a, b, e \\ Theodore Zanto $^{c}$ Triet Do ${ }^{a, b}$ Jonathan Pierce ${ }^{a, b}$ Shantanu Joshi ${ }^{a}$ \\ Ellen Woo ${ }^{a}, b \quad$ Liana G. Apostolova ${ }^{a} b, d$

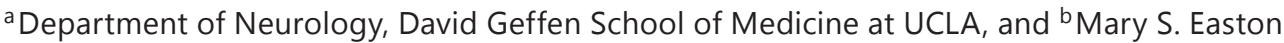 \\ Center for Alzheimer's Disease Research, Los Angeles, Calif., 'Department of Neurology, \\ UCSF, San Francisco, Calif., 'Department of Neurology, Indiana University, Indianapolis, Ind., \\ and ${ }^{e}$ Oakland University William Beaumont School of Medicine, Rochester, Mich., USA
}

\section{Key Words}

Dancing - Mild cognitive impairment - Dementia - Alzheimer's disease · Cortical thickness .

Gray matter thickness · Neuroimaging · MRI

\begin{abstract}
Introduction: We investigated the effect dance experience may have on cortical gray matter thickness and cognitive performance in elderly participants with and without mild cognitive impairment (MCI). Methods: 39 cognitively normal and $48 \mathrm{MCI}$ elderly participants completed a questionnaire regarding their lifetime experience with music, dance, and song. Participants identified themselves as either dancers or nondancers. All participants received structural 1.5-tesla MRI scans and detailed clinical and neuropsychological evaluations. An advanced 3D cortical mapping technique was then applied to calculate cortical thickness. Results: Despite having a trend-level significantly thinner cortex, dancers performed better in cognitive tasks involving learning and memory, such as the California Verbal Learning TestII (CVLT-II) short delay free recall ( $p=0.004)$, the CVLT-II long delay free recall $(p=0.003)$, and the CVLT-II learning over trials $1-5$ ( $p=0.001)$. Discussion: Together, these results suggest that dance may result in an enhancement of cognitive reserve in aging, which may help avert or delay $\mathrm{MCI}$.


Porat et al.: Dance Experience and Associations with Cortical Gray Matter Thickness in the Aging Population

\section{Introduction}

How musical performance or exercise affect the brain is widely studied in neuroscience, yet, dance - a medium that combines both music and exercise - has remained mostly unexplored. Literature suggests that positive associations exist among musical ability, cognition, and gray matter (GM) thickness [1-6]. Furthermore, musical skills are positively correlated with spatial, verbal, and emotional intelligence [7-9]. Gaser and Schlaug [2] reported that musical proficiency positively correlated with GM in young adult musicians, whereas expert musicians displayed larger GM density in motor, sensory, and parietal cortices, relative to nonmusicians. Likewise, several studies have linked aerobic and resistance exercise with thicker GM and enhancement of cognitive performance in late life [10-16]. Significantly larger hippocampal volumes and improved spatial memory were observed in a randomized controlled trial of a large elderly cohort when assigned to a 1-year aerobic exercise intervention relative to stretching $[14,15]$. Results of another randomized controlled study suggested that both resistance and aerobic exercise might delay cognitive decline in elderly populations [17]. Overall, both music experience and exercise were associated with increased GM thickness and improved cognitive function. Therefore, it may be hypothesized that dancing, which combines music and exercise, may exhibit similar benefits.

To date, few studies have evaluated the effects dance may have on either cognition or brain structure. Most of the literature surrounding dancing and neuroscience fall into physical or artistic therapies [18], where dancing is used as an exercise to help rehabilitate neurodegenerative disorders (e.g. Parkinson's disease or strokes) [19] or treat social anxieties [20]. Focusing on the elderly, Verghese et al. [21] observed social dancing to be the only physical activity correlated with a decreased risk of developing dementia.

Of the few neuroimaging studies conducted, only young cognitively normal (CN) participants were incorporated [7, 22-24] and, surprisingly, lower GM densities in expert dancers were observed relative to nondancers [22-25]. Given the reported positive correlations between musical experience or exercise and GM thickness, one might find the negative correlation described in the dance literature an anomaly, anticipating a similar positive association for dance experience and GM thickness. Researchers have interpreted these unexpected results in that expert dancers may have a reorganized brain structure allowing for uncoupling of vestibular perception and reflexes to avoid feeling dizzy or nauseous while twirling [22$24]$. Another interpretation is that cortical myelination increases as dance proficiency is developed, resulting in a relative thinning of GM [26].

With an increased focus on elderly participants in the exercise and music performance literatures, we wanted to study the effect previous exposure to dance may have on older adults who were either $\mathrm{CN}$ or had mild cognitive impairment (MCI). In the MCI state, patients typically perform poorly on cognitive tests, scoring 1.5 standard deviations (SD) below the mean but still independently caring for themselves [27]. MCI patients decline into Alzheimer's disease (AD) at a rate of 10-15\% per year, as opposed to CN adults who decline at only 1-2\% [24]. Accordingly, we were interested to see if we could find a beneficial effect that dance may have on brain structure and on cognitive performance in both CN and MCI elderly. We hypothesized that dancers will show enhanced cognitive scores relative to nondancers. Additionally, we expected to find a protective effect of dance experience on cortical thinning. 
Porat et al.: Dance Experience and Associations with Cortical Gray Matter Thickness in the Aging Population

Table 1. Demographics of dancers and nondancer participants

\begin{tabular}{llll}
\hline Variable & $\begin{array}{l}\text { Dancers } \\
(\mathrm{n}=44)\end{array}$ & $\begin{array}{l}\text { Nondancers } \\
(\mathrm{n}=43)\end{array}$ & $\begin{array}{l}\mathrm{p} \text { value, } \\
\text { ANOVA or } \chi^{2}\end{array}$ \\
\hline Age, years & $69.55 \pm 9.176$ & $70.47 \pm 8.157$ & 0.623 \\
Education, years & $16.36 \pm 2.30$ & $16.30 \pm 2.94$ & 0.914 \\
Sex (M:F, 45:42) & $21: 23$ & $24: 19$ & 0.456 \\
MMSE score & $28.43 \pm 1.81$ & $27.74 \pm 2.28$ & 0.122 \\
Diagnosis (CN:MCI, 39:48) & $24: 20$ & $15: 28$ & 0.066 \\
Dance experience, years & $3.03 \pm 7.62$ & 0 & N/A \\
\hline
\end{tabular}

Figures are means \pm SD unless indicate otherwise.

\section{Methods}

\section{Participants}

Our analyses included 87 participants (39 CN and $48 \mathrm{MCI}$; 45 males and 42 females; mean age 70 years, ranging from 51 to 90 years) previously enrolled in the Imaging and Genetic Biomarkers (ImaGene) of AD longitudinal study at the Mary S. Easton Alzheimer's Disease Center at the University of California - Los Angeles (UCLA). Demographic comparisons of the nondancer and dancer groups are given in table 1. Informed consent was administered according to the rules and regulations of the UCLA Institutional Review Board. The diagnostic evaluation consisted of a physician interview, general and neurological examination, as well as detailed neuropsychological evaluation. The diagnosis of each subject was reached by consensus among neurologists and neuropsychologists.

Participants were at least 50 years old, functionally intact and living independently. Additionally, a Mini-Mental State Examination (MMSE) score (>24), a global Clinical Dementia Rating Scale score $(<1)$, English language fluency, as well as visual and hearing abilities sufficient for detailed neuropsychological testing were required for participation. Participants with a diagnosis of dementia based on the Diagnostic and Statistical Manual of Mental Disorders, fourth edition (DSM-IV), the National Institute of Neurological Communicative Disorders and Stroke (NINCDS), or the Alzheimer's Disease and Related Disorders Association (ADRDA) criteria were excluded. Any medical, neurological, or psychiatric disorders that may have an impact on cognition (such as significant drug or alcohol use in the previous 2 years, pacemakers, claustrophobia, or contraindications to MRI scanning) were also used as exclusion criteria. Diagnosis of MCI required cognitive decline of at least 1.5 SD below age and/or education norms on at least one neuropsychological test. CN subjects were required to score within normal ranges on all cognitive exams.

\section{Music and Dance Questionnaire}

All participants completed a 30-point questionnaire assessing their personal experiences with music, song, and dance. The questionnaire was either mailed to participants to fill out at their own discretion or administered during their routine clinical visit. Participants with previous dance experience, whether distant (childhood) or current (within 5 years), were assigned to the dance group. Dance experience was classified as social and/or formal proficiency; thus, amateurs (little to no formal dance training) and experts (formal training for more than 5 years) were merged within a single dance group. About one fourth of dancers noted that they were still actively dancing within the past year. Any participant who reported having no dance experience was placed in the 'nondancer' group. 


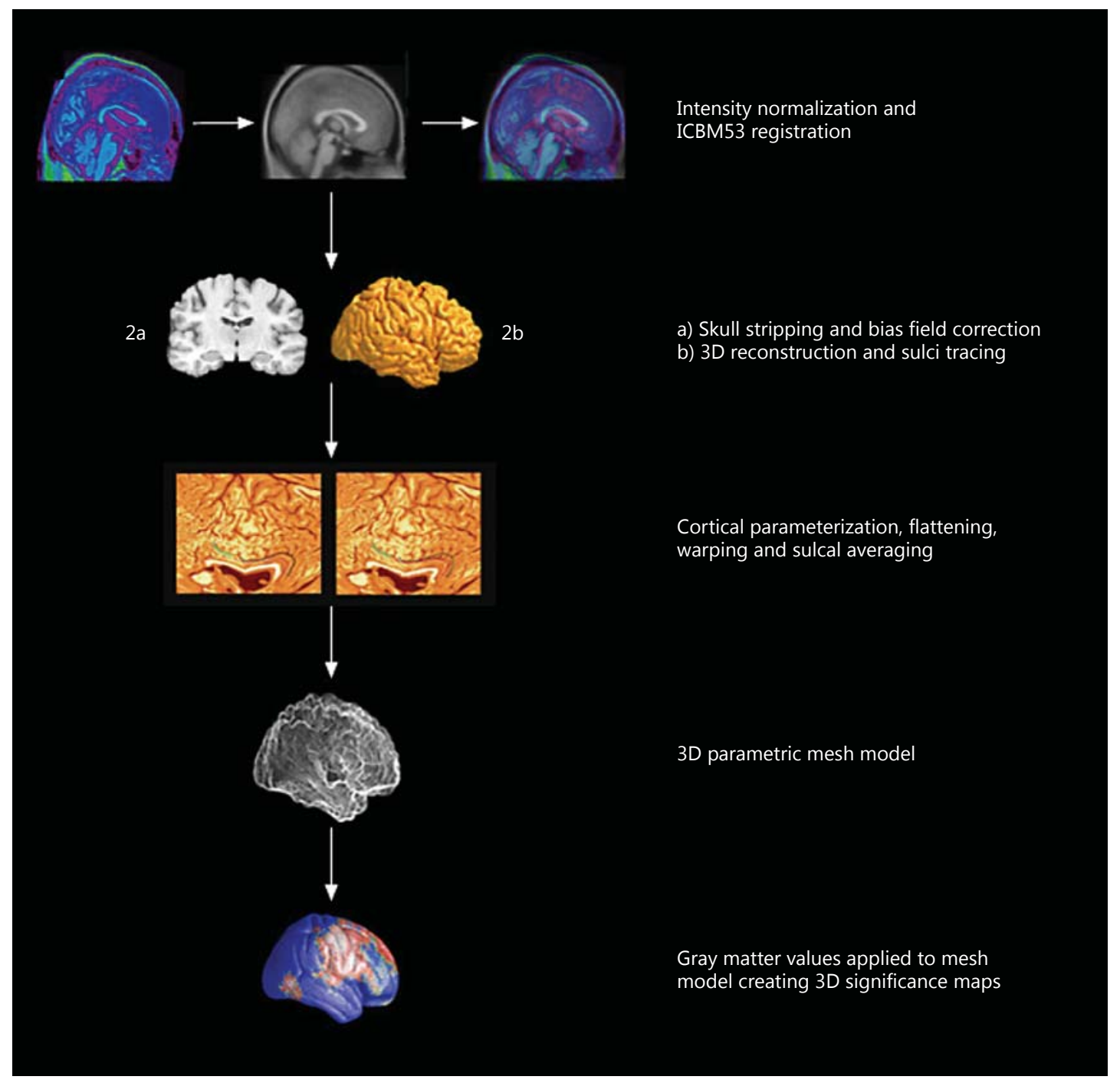

Fig. 1. Cortical thickness analysis.

\section{Imaging Acquisition}

Participants were scanned on a 1.5-tesla MRI, and T1-weighted MR data were collected with the following protocol: coronal 3D magnetization-prepared rapid acquisition gradient echo, time to recovery (TR) $28 \mathrm{~ms}$, time to echo (TE) $4.5 \mathrm{~ms}$, field of view (FOV) $22 \mathrm{~cm}$, matrix $256 \times 192$, slice/gap thickness $1.5 / 0 \mathrm{~mm}$. Additional sequences used in the determination of white matter disease burden and strokes were obtained with the following parameters: axial T2 BLADE with TR 4,000 ms, TE $99 \mathrm{~ms}$, FOV $22 \mathrm{~cm}$, matrix $320 \times 320$, and slice/gap thickness 5/1.5 mm, axial fluid-attenuated inversion recovery with TR 9,000 ms, TE $99 \mathrm{~ms}$, FOV $22 \mathrm{~cm}$, matrix $256 \times 256$, and slice/gap thickness $5 / 1.5 \mathrm{~mm}$, and axial diffusion-weighted imaging with TR 7,000 ms, TE $123 \mathrm{~ms}$, FOV $22 \mathrm{~cm}$, matrix 128, propeller correction, and slice/gap thickness $5 / 1.5 \mathrm{~mm}$.

\section{Cortical Thickness Analysis}

Figure 1 illustrates the steps of our imaging methodology, which were described in depth in previous publications [28]. First, MRI scans were spatially normalized to the International 
Consortium for Brain Mapping 53 (ICBM53) average brain imaging template and subjected to intensity normalization [24]. We then used the software BrainSuite for automated masking of the 'whole brain' [29]. Manual inspection and editing of the 'whole brain' mask was then carried out to exclude any nonbrain tissue, the brainstem, and the cerebellum, as well as to split the mask into left and right hemispheres. 3D hemispheric reconstructions were then created to allow an experienced researcher to trace 38 sulci per hemisphere following an extensive validated tracing protocol [30]. The sulcal lines for all participants were used to create a study-specific average sulcal map which was then used as a target for spatial alignment of the individual sulcal lines, and hence each subject's unique cortical morphology. This was achieved by parameterizing and warping of the flattened cortical surfaces as described in Thompson et al. [31]. The end result is a more precisely matched cortical morphology with improved signal-to-noise ratio [24]. We applied BrainSuite's partial volume classifier [25] to the spatially and intensity-corrected MRI data in order to obtain GM, white matter, and cerebrospinal fluid tissue segmentations. The GM was then resampled to $0.33 \mathrm{~mm}$ isotropic voxel size in order to obtain an index GM thickness at subvoxel spatial resolution [27]. Next we calculated GM thickness at each surface point for each individual participant as the shortest distance between GM to white matter and GM to cerebrospinal fluid interfaces. The thickness values were then mapped onto the cortical surface models and subjected to linear regression analyses as described previously [27]. Our final results are displayed on 3D significance maps, seen as the final result in figure 1.

\section{Statistical Analyses}

We used independent-sample $t$ tests and $\chi^{2}$ tests as appropriate to compare the demographic variables between groups. Linear regression models were used to study the effects of dancing on GM thickness while correcting for diagnosis. Linear regressions also assessed the relationship between cortical thickness and neuropsychological examination scores in dancers and nondancers correcting for age. To correct for multiple comparisons, we ran 100,000 permutations using $\mathrm{p}<0.01$ significance level thresholds as reported previously by Apostolova et al. [32]. The predictor variable was permuted across our sample 100,000 times. The final corrected $p$ value reflects the proportion of the 100,000 runs that produced a $p$ value that 'beats' the original $p$ value. Our corrected $p$ value of 0.01 would mean that only $1 \%$ of the permutation runs resulted in a more significant $p$ value than the one we observed in the true experiment.

\section{Results}

\section{Demographics}

Demographic comparisons of the nondancer and dancer groups can be seen in table 1 . There were no significant differences between the groups in age, sex, education, or MMSE score. A trend-level difference was seen in regard to diagnosis with the nondancer group having a higher proportion of MCI subjects relative to the dancer group (65 vs. $45 \%, \mathrm{p}=$ $0.066)$.

\section{Neuropsychological Data}

Table 2 includes the mean and SD of neuropsychological scores for both groups, showing that dancers performed better than nondancers in learning and memory [California Verbal Learning Test-II (CVLT-II) number of words learned across trials $1-5, \mathrm{p}=0.001$, CVLT-II short delay free recall, $p=0.004$, CVLT-II long delay free recall, $p=0.003$ ]. Due to absent CVLT-II scores, 2 participants from the dancer group were excluded from this analysis. 
Table 2. Dancers and nondancers CVLT-II comparison

\begin{tabular}{lcccc}
\hline Variable & Dancers & Nondancers & $\begin{array}{c}\text { Student's } \\
\text { t statistic }\end{array}$ & p value \\
\hline Learning over trials 1 -5 & $46.85 \pm 12.49$ & $37.69 \pm 11.7$ & -3.48 & 0.001 \\
Short delay free recall & $9.6 \pm 4.3$ & $6.9 \pm 4.1$ & -3.00 & 0.004 \\
Long delay free recall & $10.0 \pm 5.0$ & $7.0 \pm 4.0$ & -3.11 & 0.003 \\
\hline
\end{tabular}

Figures are means \pm SD unless indicated otherwise.

Fig. 2. 3D significance maps comparing cortical thickness between nondancers and dancers, corrected for diagnosis. Here, dancers show significantly less GM in the red and white areas. Colors refer to the online version only.

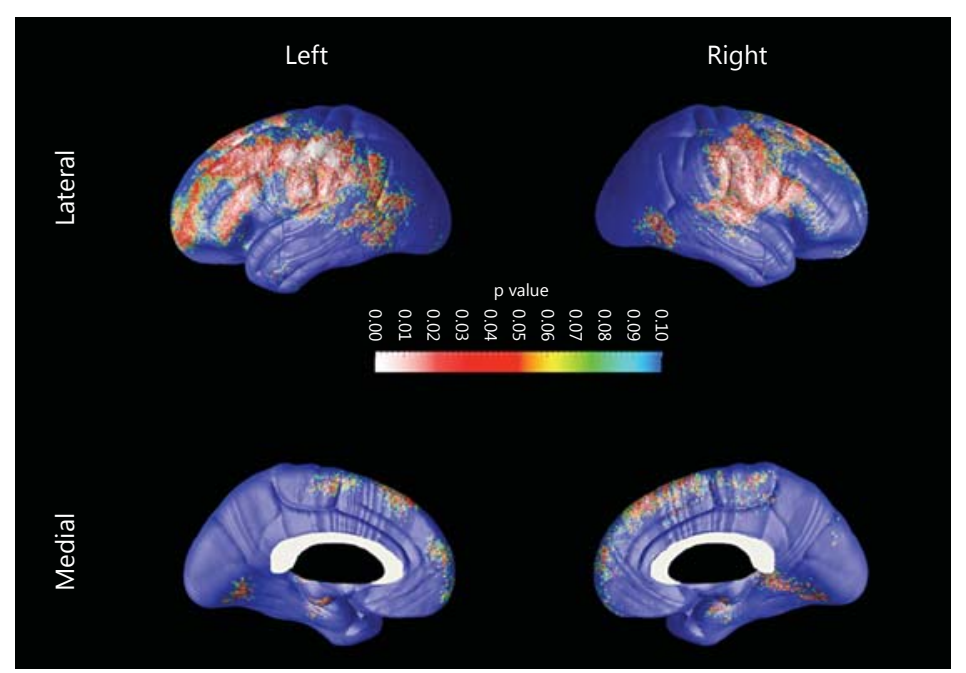

\section{Cortical Thickness Analyses}

As MCI diagnosis was relatively more common in nondancers compared to dancers $(\mathrm{p}=$ 0.066 ) in our sample, we elected to correct for diagnosis in our analyses. Dancers showed trend-level thinner GM relative to nondancers in the primary motor, somatosensory and prefrontal cortical regions predominantly on the left side (right $\mathrm{p}_{\text {corrected }}=0.066$, left $\mathrm{p}_{\text {corrected }}=0.057$; fig. 2). Next, we investigated the association between CVLT-II performance and cortical thickness in each group while correcting for age (fig. 3). We found significant associations between cognitive performance and cortical thickness in nondancers only. There were significant positive associations between cortical thickness with learning across CVLT-II trials 1-5 (left $\mathrm{p}_{\text {corrected }}=0.09$, right $\mathrm{p}_{\text {corrected }}=0.05$ ) and CVLT-II short delay free recall (left $\mathrm{p}_{\text {corrected }}=0.017$, right $\mathrm{p}_{\text {corrected }}=0.048$ ) bilaterally, as well as trend-level significant association with CVLT-II long delay free recall on the right (left $\mathrm{p}_{\text {corrected }}=0.14$, right $\mathrm{p}_{\text {corrected }}=$ 0.08). These findings remained unchanged after we corrected for diagnosis.

\section{Discussion}

We observed thinner cortical GM bilaterally in dancers relative to nondancers. Additionally, dancers outperformed nondancers in learning and memory tasks. Our findings are similar to previous studies where young adults showed thinner cortical GM in dancers relative to nondancers [18-21]. The associations between dance experience and GM thickness in the 


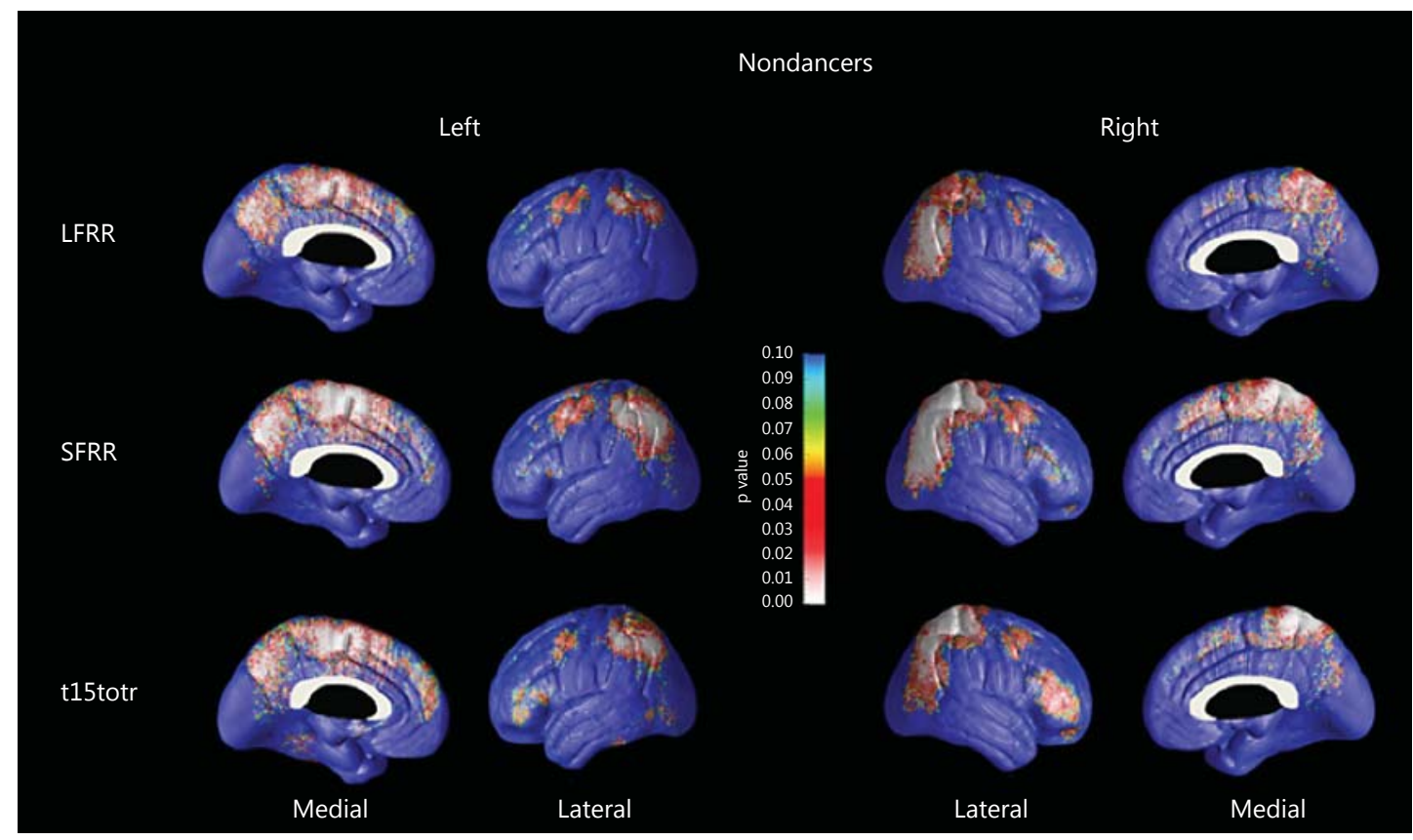

Fig. 3. 3D significance maps (corrected for age) of the association between cortical thickness and long/short delay free recall (LFRR, SFRR), and CVLT-II trials 1-5 (t15totr) neurocognitive scores. Higher scores were associated with thicker GM volumes in nondancers.

elderly have not, to our knowledge, been investigated previously. Interestingly, only the nondancer group showed typical patterns of association between cognitive decline and GM atrophy commonly seen in MCI patients who decline into AD [32-36]. Other labs, as well as ours, have previously identified that $\mathrm{CN}$ and MCI patients who are prone to develop AD show a typical pattern of cortical atrophy with prominent involvement of the entorhinal, inferior and lateral temporal cortices, as well as the lateral and medial parietal and posterior cingulate cortices. The frontal cortex is normally spared until later in the disease course [37, 38]. The pattern of correlation between cognitive performance and cortical thickness in the nondancer group (fig. 3) shows a significant resemblance to the atrophy pattern seen in patients suffering from $\mathrm{AD}[39,40]$. Our findings suggest that dance experience during life may enhance cognitive reserve as previously suggested for exercise and music [41-45].

Kattenstroth et al. [46, 47] in 2010 and 2013 conducted studies with dance as an intervention to combat postural and cognitive declines in elderly cohorts, as well as assessments of cognition in relation to previous dance experience. They observed in both studies that those assigned to the dancer group displayed enhanced cognitive abilities as well as postural and gait benefits in relation to nondancing controls.

Yaakov Stern's [48] research describes how the brain employs 'cognitive reserve' by using preexisting cognitive processing approaches and compensation mechanisms to counterbalance for structural damage. Subjects with cognitive reserve are said to display structural brain atrophy without (or with minimal) clinical symptoms. Despite having thinner GM, dancers excelled in learning and memory tasks. The absence of association between memory performance and cortical thickness in dancers is highly suggestive of cognitive reserve in dancers, especially since the association was observed in the nondancer group.

The greatest limitation of our study is the subjective classification of dancer versus nondancer, as all of our participants self-asserted their dancing exposure amount and profi- 
Porat et al.: Dance Experience and Associations with Cortical Gray Matter Thickness in the Aging Population

ciency levels. Given our study design, we gathered data from surveys, and unfortunately do not have more information than what we present in this paper. Future studies with more finegrained skill level assessment will be needed to confirm our findings. Additionally, due to the small sample size of dancers, we were unable to stratify dancers by skill level - i.e., examine the effects of dance on cortical thickness and cognition separately in experts versus amateurs. While this study is cross-sectional, we are collecting longitudinal data on these participants. Future investigation of the effect dance experience has on cognitive decline, conversion to dementia, as well as longitudinal rates of cortical and hippocampal atrophy may be observed. Lastly, as other studies demonstrated the positive effects either music or exercise have on health [49-53], it would be very informative to study the effect dance-based therapeutic programs have on participants with cognitive and structural brain decline.

Overall, we determined dancers to have thinner cortical GM relative to nondancers in frontal brain regions. Our findings align well with previously published research [18-21]. Despite having thinner cortex in some cortical areas, the dancer group manifested superior memory performance and absence of the expected association between cognitive decline and cortical atrophy in AD-relevant cortical areas, an association that was readily observed among nondancers. This observation could represent evidence of cognitive reserve conferred by dance activities earlier in life.

\section{Acknowledgments}

We would like to thank all the participants and researchers involved in this project. Our work was generously supported by NIA R01 AG040770; NIA K02 AG048240 Easton Consortium for Alzheimer Drug Discovery and Biomarker Development, and the NIA P50 AG16570.

\section{References}

1 Rauscher FH, Shaw GL, Ky KN: Music and spatial task performance. Nature 1993;365:611.

-2 Gaser C, Schlaug G: Brain structures differ between musicians and non-musicians. J Neurosci 2003;23:92409245.

-3 Angelucci F, Fiore M, Ricci E, Padua L, Sabino A, Tonali PA: Investigating the neurobiology of music: brainderived neurotrophic factor modulation in the hippocampus of young adult mice. Behav Pharmacol 2007;18: 491-496.

4 Hyde KL, Lerch J, Norton A, Forgeard M, Winner E, Evans AC, et al: Musical training shapes structural brain development. J Neurosci 2009;29:3019-3025.

5 Groussard M, La Joie R, Rauchs G, Landeau B, Chetelat G, Viader F, et al: When music and long-term memory interact: effects of musical expertise on functional and structural plasticity in the hippocampus. PLoS One 2010;5:e13225.

6 Groussard M, Viader F, Hubert V, Landeau B, Abbas A, Desgranges B, et al: Musical and verbal semantic memory: two distinct neural networks? Neuroimage 2010;49:2764-2773.

7 Schellenberg EG, Hallam S: Music listening and cognitive abilities in 10- and 11-year-olds: the blur effect. Ann NY Acad Sci 2005;1060:202-209.

-8 Forgeard M, Winner E, Norton A, Schlaug G: Practicing a musical instrument in childhood is associated with enhanced verbal ability and nonverbal reasoning. PLoS One 2008;3:e3566.

9 Nair BR, Browne W, Marley J, Heim C: Music and dementia. Degener Neurol Neuromuscul Dis 2013;3:47-51.

$\checkmark 10$ Erickson KI, Raji CA, Lopez OL, Becker JT, Rosano C, Newman AB, et al: Physical activity predicts gray matter volume in late adulthood: the Cardiovascular Health Study. Neurology 2010;75:1415-1422.

11 Liu-Ambrose T, Nagamatsu LS, Graf P, Beattie BL, Ashe MC, Handy TC: Resistance training and executive functions: a 12-month randomized controlled trial. Arch Intern Med 2010;170:170-178.

12 Profenno LA, Porsteinsson AP, Faraone SV: Meta-analysis of Alzheimer's disease risk with obesity, diabetes, and related disorders. Biol Psychiatry 2010;67:505-512.

13 Ruscheweyh R, Willemer C, Kruger K, Duning T, Warnecke T, Sommer J, et al: Physical activity and memory functions: an interventional study. Neurobiol Aging 2011;32:1304-1319. 
Porat et al.: Dance Experience and Associations with Cortical Gray Matter Thickness in the Aging Population

14 Siervo M, Arnold R, Wells JC, Tagliabue A, Colantuoni A, Albanese E, et al: Intentional weight loss in overweight and obese individuals and cognitive function: a systematic review and meta-analysis. Obes Rev 2011;12:968983.

15 Ahlskog JE, Geda YE, Graff-Radford NR, Petersen RC: Physical exercise as a preventive or disease-modifying treatment of dementia and brain aging. Mayo Clin Proc 2011;86:876-884.

16 Mortimer JA, Ding D, Borenstein AR, DeCarli C, Guo Q, Wu Y, et al: Changes in brain volume and cognition in a randomized trial of exercise and social interaction in a community-based sample of non-demented Chinese elders. J Alzheimers Dis 2012;30:757-766.

17 Yaffe K, Hoang T: Nonpharmacologic treatment and prevention strategies for dementia. Continuum (Minneap Minn) 2013;19:372-381.

18 Noice T, Noice H, Kramer AF: Participatory arts for older adults: a review of benefits and challenges. Gerontologist 2014;54:741-753.

19 McGill A, Houston S, Lee RY: Dance for Parkinson's: a new framework for research on its physical, mental, emotional, and social benefits. Complement Ther Med 2014;22:426-432.

20 Tavormina R, Tavormina MG, Nemoianni E: 'Dance and go on': a project of psychosocial rehabilitation on the road. Psychiatr Danub 2015;27(suppl 1):S143-S147.

21 Verghese J, Lipton RB, Katz MJ, Hall CB, Derby CA, Kuslansky G, et al: Leisure activities and the risk of dementia in the elderly. N Engl J Med 2003;348:2508-2516.

-22 Hanggi J, Koeneke S, Bezzola L, Jancke L: Structural neuroplasticity in the sensorimotor network of professional female ballet dancers. Hum Brain Mapp 2010;31:1196-1206.

23 Nigmatullina Y, Hellyer PJ, Nachev P, Sharp DJ, Seemungal BM: The neuroanatomical correlates of trainingrelated perceptuo-reflex uncoupling in dancers. Cereb Cortex 2015;25:554-562.

24 Hufner K, Binetti C, Hamilton DA, Stephan T, Flanagin VL, Linn J, et al: Structural and functional plasticity of the hippocampal formation in professional dancers and slackliners. Hippocampus 2011;21:855-865.

25 Brown S, Martinez MJ, Parsons LM: The neural basis of human dance. Cereb Cortex 2006;16:1157-1167.

26 Draganski B, Gaser C, Busch V, Schuierer G, Bogdahn U, May A: Neuroplasticity: changes in grey matter induced by training. Nature 2004;427:311-312.

27 Petersen RC, Doody R, Kurz A, Mohs RC, Morris JC, Rabins PV, et al: Current concepts in mild cognitive impairment. Arch Neurol 2001;58:1985-1992.

-28 Apostolova LG, Steiner CA, Akopyan GG, Dutton RA, Hayashi KM, Toga AW, et al: Three-dimensional gray matter atrophy mapping in mild cognitive impairment and mild Alzheimer disease. Arch Neurol 2007;64: 1489-1495.

29 Shattuck DW, Leahy RM: BrainSuite: an automated cortical surface identification tool. Med Image Anal 2002; 6:129-142.

30 Sowell E, Mega M, Zoumalan C, Lindshield C, Rex D: Gyral pattern delineation in 3D: surface curve protocol. 2000. http://www.loni.ucla.edu/ esowell/new_sulcvar.html.

-31 Thompson PM, Hayashi KM, Sowell ER, Gogtay N, Giedd JN, Rapoport JL, et al: Mapping cortical change in Alzheimer's disease, brain development, and schizophrenia. Neuroimage 2004;23(suppl 1):S2-S18.

-32 Apostolova LG, Hwang KS, Medina LD, Green AE, Braskie MN, Dutton RA, et al: Cortical and hippocampal atrophy in patients with autosomal dominant familial Alzheimer's disease. Dement Geriatr Cogn Disord 2011; 32:118-125.

-33 Apostolova LG, Dutton RA, Dinov ID, Hayashi KM, Toga AW, Cummings JL, et al: Conversion of mild cognitive impairment to Alzheimer disease predicted by hippocampal atrophy maps. Arch Neurol 2006;63:693-699.

-34 Thompson PM, Hayashi KM, de Zubicaray G, Janke AL, Rose SE, Semple J, et al: Dynamics of gray matter loss in Alzheimer's disease. J Neurosci 2003;23:994-1005.

35 Bozzali M, Filippi M, Magnani G, Cercignani M, Franceschi M, Schiatti E, et al: The contribution of voxel-based morphometry in staging patients with mild cognitive impairment. Neurology 2006;67:453-460.

-36 Chetelat G, Desgranges B, De La Sayette V, Viader F, Eustache F, Baron JC: Mapping gray matter loss with voxelbased morphometry in mild cognitive impairment. Neuroreport 2002;13:1939-1943.

-37 Apostolova LG, Dinov ID, Dutton RA, Hayashi KM, Toga AW, Cummings JL, et al: 3D comparison of hippocampal atrophy in amnestic mild cognitive impairment and Alzheimer's disease. Brain 2006;129(Pt 11):2867-2873.

38 Apostolova LG, Mosconi L, Thompson PM, Green AE, Hwang KS, Ramirez A, et al: Subregional hippocampal atrophy predicts Alzheimer's dementia in the cognitively normal. Neurobiol Aging 2010;31:1077-1088.

-39 Apostolova LG, Thompson PM, Green AE, Hwang KS, Zoumalan C, Jack CR Jr, et al: 3D comparison of low, intermediate, and advanced hippocampal atrophy in MCI. Hum Brain Mapp 2010;31:786-797.

40 Apostolova LG, Thompson PM: Mapping progressive brain structural changes in early Alzheimer's disease and mild cognitive impairment. Neuropsychologia 2008;46:1597-1612.

41 Duarte A, Hayasaka S, Du A, Schuff N, Jahng GH, Kramer J, et al: Volumetric correlates of memory and executive function in normal elderly, mild cognitive impairment and Alzheimer's disease. Neurosci Lett 2006;406: 60-65.

42 Vance DE, Crowe M: A proposed model of neuroplasticity and cognitive reserve in older adults. Act Adapt Aging 2006;30:61-79.

43 Hanna-Pladdy B, MacKay A: The relation between instrumental musical activity and cognitive aging. Neuropsychology 2011;25:378-386.

44 Scarmeas N, Stern Y: Cognitive reserve and lifestyle. J Clin Exp Neuropsychol 2003;25:625-633. 
45 Whalley LJ, Deary IJ, Appleton CL, Starr JM: Cognitive reserve and the neurobiology of cognitive aging. Ageing Res Rev 2004;3:369-382.

46 Kattenstroth JC, Kolankowska I, Kalisch T, Dinse HR: Superior sensory, motor, and cognitive performance in elderly individuals with multi-year dancing activities. Front Aging Neurosci 2010;2:31.

47 Kattenstroth JC, Kalisch T, Holt S, Tegenthoff M, Dinse HR: Six months of dance intervention enhances postural, sensorimotor, and cognitive performance in elderly without affecting cardio-respiratory functions. Front Aging Neurosci 2013;5:5.

48 Stern Y: Cognitive reserve in ageing and Alzheimer's disease. Lancet Neurol 2012;11:1006-1012.

-49 Carrion C, Aymerich M, Bailles E, Lopez-Bermejo A: Cognitive psychosocial intervention in dementia: a systematic review. Dement Geriatr Cogn Disord 2013;36:363-375.

50 Karp A, Paillard-Borg S, Wang HX, Silverstein M, Winblad B, Fratiglioni L: Mental, physical and social components in leisure activities equally contribute to decrease dementia risk. Dement Geriatr Cogn Disord 2006;21: 65-73.

51 Ohman H, Savikko N, Strandberg TE, Pitkala KH: Effect of physical exercise on cognitive performance in older adults with mild cognitive impairment or dementia: a systematic review. Dement Geriatr Cogn Disord 2014; 38:347-365.

52 Kemoun G, Thibaud M, Roumagne N, Carette P, Albinet C, Toussaint L, et al: Effects of a physical training programme on cognitive function and walking efficiency in elderly persons with dementia. Dement Geriatr Cogn Disord 2010;29:109-114.

53 Irish M, Cunningham CJ, Walsh JB, Coakley D, Lawlor BA, Robertson IH, et al: Investigating the enhancing effect of music on autobiographical memory in mild Alzheimer's disease. Dement Geriatr Cogn Disord 2006;22: 108-120. 